\title{
ORIGINAL RESEARCH \\ Diffusion Tensor Group Tractography of the Corpus Callosum in Clinically Isolated Syndrome
}

F. Lin

C. Yu

Y. Liu

K. Li

H. Lei

BACKGROUND AND PURPOSE: Many studies have observed atrophy and abnormal diffusion within the CC in MS. However, few studies have addressed whether such abnormalities appear at the earliest stage of MS, especially in CIS. In this study, we aimed to investigate the CC integrity and patterns of $\mathrm{CC}$ abnormalities in $\mathrm{CIS}$ with diffusion tensor group tractography.

MATERIALS AND METHODS: First, probability maps of the entire CC and its subregions (genu, body, and splenium) were created from 19 healthy subjects. Then these probability maps were used to evaluate diffusion within the entire CC and its segments in 19 patients with $\mathrm{CIS}$. Five indices, including the midsagittal CC area, FA, MD, $\lambda_{1}$, and $\lambda_{23}$, were used to characterize CC integrity

RESULTS: Significant differences were found between patients with CIS and healthy controls in the entire CC and its segments. For the entire CC, patients with CIS had a significantly lower midsagittal $\mathrm{CC}$ area and FA, higher MD and $\lambda_{23}$, with a trend toward higher $\lambda_{1}$. These 4 diffusion measures were correlated with T2 lesion volume. Moreover, abnormal white matter integrity was present in subregions of the CC; there was a robust significant increase in $\lambda_{23}$ in the body and splenium and no difference in $\lambda_{1}$ in the genu.

CONCLUSIONS: Our results suggest that atrophy and abnormal diffusion inside the CC appear at the stage of $\mathrm{CIS}$ and the severity of damage in the genu is milder than that in the body and splenium.

ABBREVIATIONS: $\mathrm{CC}=$ corpus callosum; $\mathrm{CIS}=$ clinically isolated syndrome; $\mathrm{DTI}=$ diffusion tensor imaging; EDSS = Expanded Disability Status Scale; FA = fractional anisotropy; FACT = fiber assignment by continuous tracking; FLAIR = fluid-attenuated inversion recovery; $\lambda_{1}=$ axial diffusivity; $\lambda_{23}=$ radial diffusivity; $\mathrm{MD}=$ mean diffusivity; $\mathrm{MNI}=$ Montreal Neurological Institute; $\mathrm{MS}=$ multiple sclerosis; NACC = normal-appearing corpus callosum; NAWM = normal-appearing white matter; $\mathrm{NC}=$ healthy controls; TSE $=$ turbo spin-echo

A trophy of the CC, the largest white matter fiber bundle linking the 2 hemispheres, is commonly observed in clinically definite MS by using conventional MR imaging. ${ }^{1-3} \mathrm{Si}-$ mon et $\mathrm{al}^{2}$ found that patients with MS had a significantly lower midsagittal CC area compared with that in healthy controls. Dietemann et $\mathrm{al}^{3}$ also reported that severe atrophy of the CC occurs in longstanding MS and that patients with severe CC atrophy present serious clinical symptoms. Abnormal CC integrity (reduced FA or increased MD) is also a common finding in MS using DTI. ${ }^{4-8}$ Moreover, some DTI studies have found different change patterns in the subdivisions of the CC.

Received April 22, 2010; accepted after revision June 16

From the State Key Laboratory of Magnetic Resonance and Atomic and Molecular Physics (F.L., H.L.), Wuhan Institute of Physics and Mathematics, Chinese Academy of Sciences, Wuhan, People's Republic of China; Department of Radiology (C.Y.), Tianjin Medical University General Hospital, Tianjin, People's Republic of China; and Department of Radiology (C.Y., Y.L., K.L.), Xuanwu Hospital of Capital Medical University, Beijing, People's Republic of China.

This work was partially supported by the Natural Science Foundation of China (30800252, 30670601, 30470519, 30930029 and 20921004), 973 Program of the Ministry of Science and Technology of China (2011CB707800), the Beijing Scientific and Technological New Star Program (2005B21), the Knowledge Innovation Program of the Chinese Academy of Sciences, and the Foundation of President of The Chinese Academy of Sciences.

Please address correspondence to Fuchun Lin, PhD, State Key Laboratory of Magnetic Resonance and Atomic and Molecular Physics, Wuhan Institute of Physics and Mathematics, Chinese Academy of Sciences, Wuhan 430071, People's Republic of China; e-mail: fclin@wipm.ac.cn

Indicates open access to non-subscribers at www.ajnr.org

DOI 10.3174/ajnr.A2273
Some authors observed FA decreases predominantly in the body of the $\mathrm{CC},{ }^{6-8}$ with an insignificant trend in the splenium, ${ }^{7,8}$ and no significant changes in the genu. ${ }^{7}$ Therefore, some researchers reported that CC abnormalities might be a sensitive marker of MS., ${ }^{9,10}$

CIS, suggestive of MS, as typically the earliest clinical expression of many patients with MS, ${ }^{11}$ has received a great deal of attention from recent clinical research. Studies on CIS may provide new insights into early pathologic changes and pathogenetic mechanisms that might affect the course of the disorder. ${ }^{12}$ Nevertheless, to our knowledge, there are few studies concerning the onset of CC atrophy, especially at the stage of CIS. ${ }^{13,14}$ Bester et $\mathrm{al}^{13}$ found abnormal diffusion within the NACC in patients with CIS with optic neuritis on the basis of region-of-interest analysis. Their findings suggested that the $\mathrm{CC}$ is an early site for the development of anisotropic changes in MS. $^{13}$ Another study using histogram analysis demonstrated that occult damage occurs in normal-appearing brain tissue in CIS and the occult damage might be related to both T2 lesion load and brain tissue atrophy. ${ }^{14}$ They also found that some DTI histogram measures might be useful for assessing the disease progression in patients with CIS and MS. ${ }^{14}$

The above-mentioned articles suggested that the brain tissue might be damaged in CIS; however, to our knowledge, there is no study of CC integrity and its damage patterns. Therefore, in the present study, we first examined CC integrity at the stage of CIS with diffusion tensor group tractography, ${ }^{15,16}$ which can avoid unreliable and erroneous termination in fiber tracking because of the extremely low FA value in the NAWM in diseased brains, especially in the lesions. ${ }^{17,18}$ 
This method is also capable of constructing the probability maps of fiber tracts on the basis of healthy DTI datasets; the probability maps can be used as reference template images to study abnormal diffusion within the tracts in neurodegenerative diseases. ${ }^{15,16,19}$ Moreover, the fiber distribution in the CC is known to be heterogeneous, ${ }^{20-22}$ and there are different change patterns in the subdivisions of the CC in $\mathrm{MS}^{6-8}$; therefore, we hypothesized that the patterns of CC abnormalities are also spatially heterogeneous in CIS. To show this heterogeneity, we parcellated the entire CC further into 3 subregions on the basis of known callosal anatomic projections, which are the genu, body, and splenium ${ }^{23-26}$; then, we created their probability maps and used them to evaluate the diffusion indices inside these regions. Finally, statistical analysis and correlation analysis were performed.

\section{Materials and Methods}

\section{Subjects}

In this study, 19 patients with CIS (10 women), 4 with optic neuritis, 3 with brain stem syndromes, 10 with spinal cord syndromes, and 2 with multifocal abnormalities, were prospectively examined according to the following criteria $^{11}: 1$ ) a single clinical episode suggestive of MS with at least 1 visible brain lesion on T2-weighted images; 2) presentation within 6 months from onset; 3) no corticosteroid or immunosuppressant treatment for at least 3 months before scanning; 4) exclusion of other possible diagnoses; and 5) no brain imaging artifacts. Their mean age was $39.5 \pm 11.6$ years (range, 19.0-60.0 years), the mean duration of the disease was $47 \pm 55$ days (range, $1-150$ days), and the mean EDSS score ${ }^{27}$ was $2.8 \pm 1.9$ (range, $1.0-$ 8.5). Nineteen age- and sex-matched healthy subjects (10 women; mean age, $38.9 \pm 11.0$ years; range, $18.0-58.0$ years) with no history of neurologic or psychopathic disorders and normal neurologic examination findings, were selected as healthy controls. Two neuroradiologists (C.Y., with 13 years' experience in MR image evaluation, and Y.L., with 6 years of experience) and 1 neurologist (K.L., with 18 years' experience in neurology) assigned final clinical diagnoses for all patients in consensus by reviewing all case histories, physical examination results, MR images, and laboratory data. One expert (F.L., with 7 years' experience in DTI image analysis) analyzed the DTI data. The local ethics committee of Xuanwu Hospital approved this study, and written informed consent about all aspects of the project from all participants was obtained before the MR imaging examinations.

\section{MR Imaging Data Acquisition}

MR images for all subjects were obtained with a 1.5T scanner (Sonata; Siemens, Erlangen, Germany). We used the following sequences with identical axial section positions, number of sections, ${ }^{28}$ section thicknesses (4 mm), and intersection gaps $(0.4 \mathrm{~mm}): 1)$ TSE T2-weighted imaging $(\mathrm{TR}=5500 \mathrm{~ms}, \mathrm{TE}=94 \mathrm{~ms}, \mathrm{NEX}=3$, echo-train length $=$ 11 , matrix $=256 \times 224$, FOV $=240 \times 210 \mathrm{~mm}^{2}$ ), 2) a FLAIR sequence $(\mathrm{TR}=8500 \mathrm{~ms}, \mathrm{TE}=150 \mathrm{~ms}, \mathrm{NEX}=1, \mathrm{TI}=2200 \mathrm{~ms}$, echo-train length $=8$, matrix $\left.=256 \times 224, \mathrm{FOV}=240 \times 210 \mathrm{~mm}^{2}\right)$, and 3 ) a spin-echo single-shot echo-planar pulse sequence $(\mathrm{TR}=$ $5000 \mathrm{~ms}, \mathrm{TE}=100 \mathrm{~ms}, \mathrm{NEX}=10$, matrix $=128 \times 112$ zero-filled into $256 \times 224, \mathrm{FOV}=240 \times 210 \mathrm{~mm}^{2}$ ). We obtained a total of 7 image sets: 6 with noncollinear diffusion-weighting gradients and a b-value of $1000 \mathrm{~s} / \mathrm{mm}^{2}$ and 1 without diffusion-weighting.

\section{Data Preprocessing}

The visible lesions were identified on the TSE T2-weighted and FLAIR images and were manually extracted by an experienced radiologist by using MRIcro (http://www.cabiatl.com/mricro). Then the T2 lesion volume of each patient was calculated. For DTI data, all diffusionweighted images were first visually inspected for apparent artifacts arising from subject motion and instrument malfunction, and then distortions caused by eddy currents and simple head motions were corrected by the Functional MRI of the Brain Diffusion Toolbox (FSL; http://www.fmrib.ox.ac.uk/fsl). Finally, the DTI data were interpolated into isotropic voxels of $0.94 \mathrm{~mm}$.

\section{Diffusion Indices}

The diffusion tensor elements for each voxel were first estimated with the multivariate linear fitting algorithm ${ }^{29}$; then, diffusion tensor was diagonalized to obtain its 3 pairs of eigenvalues $\left(\lambda_{1}, \lambda_{2}\right.$, and $\left.\lambda_{3}\right)$ and eigenvectors. To quantitatively characterize the CC integrity, we generated 4 indices from these eigenvalues: FA measuring the degree of diffusion anisotropy; MD measuring the magnitude of average diffusion; $\lambda_{1}$ measuring the magnitude of diffusivity parallel to the direction of maximum diffusion; and $\lambda_{23}$, the average value of $\lambda_{2}$ and $\lambda_{3}$, measuring the magnitude of diffusivity perpendicular to the direction of maximum diffusion. ${ }^{28,30}$

\section{Size}

In the present study, the size of the CC was defined as the area of the structure seen on the midsagittal plane of the reconstructed FAweighted color-coded images, because the outline of the CC in this plane was red and easily identified. Moreover, this region of interest was expected to be reproducible across subjects. Each of the marginal pixels of the region of interest was identified when it was located at the margin of the CC and showed high red intensity on the FA-weighted color-coded images.

\section{Fiber Tractography}

The tractography for the CC was implemented with DTIStudio (Johns Hopkins University, Baltimore, Maryland), ${ }^{31}$ in which the FACT method was used. ${ }^{32}$ Fiber tracking was performed from all voxels with an FA value of $>0.25$ inside the brain, according to the FA threshold of $0.25-0.35$ for fiber reconstruction recommended by Mori et $\mathrm{al}^{33}$ and Stieltjes et $\mathrm{al}^{34}$ and was terminated when the FA value was $<0.2$ or the trajectory angle between 2 connected voxels was $>40^{\circ}$. To reconstruct the CC, the midsagittal plane on the FAweighted color-coded images was used as the filter region of interest and fibers passing through this filtered region of interest were taken as the entire CC. ${ }^{35,36}$ To evaluate the patterns of CC abnormalities in patients with CIS, we evenly divided the entire CC on the FAweighted color-coded image into 4 quadrants along its anteroposterior axis, ${ }^{25}$ which roughly corresponded to the genu, anterior and posterior part of truncus (body), and the splenium. ${ }^{23-26}$ One example of the 3 subregions of the CC is shown in Fig $1 A$.

\section{Probabilistic Map}

Tractography is less reliable in lesions of diseased subjects than in healthy controls because it is affected by the altered DTI parameters, ${ }^{17,18}$ which may cause the erroneous termination of fiber tracking and influence reliabilities between group comparisons. Therefore, we first created the entire CC and its subregion probability maps from healthy volunteer DTI datasets and then applied these probability maps as weighted images to evaluate diffusion indices inside the 

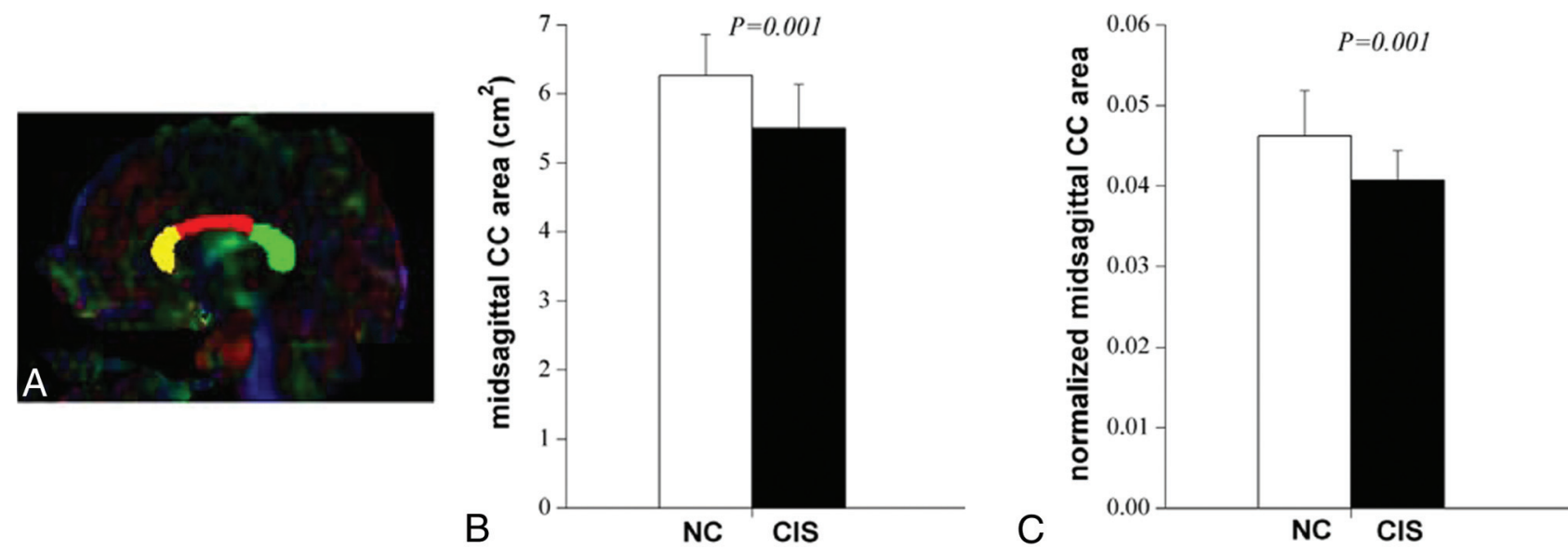

Fig 1. The size of the midsagittal CC for NC and CIS suggestive of MS groups. A, One example of midsagittal CC and its subregions (yellow indicates the genu; red, the body; green, the splenium). $B$, The midsagittal CC area for the 2 groups. $C$, The normalized midsagittal CC area by midsagittal brain for the 2 groups.

tract. ${ }^{15,16,19}$ The CC probability map was constructed as follows: First, the entire CC was reconstructed by FACT for each healthy subject, and then the diffusion unweighted $(b=0)$ image was normalized to the standard MNI space by using an echo-planar imaging template with Statistical Parametric Mapping 2 (http://www.fil.ion.ucl.ac.uk/ spm). Then, the transformation parameter was applied to the fiber tracts to normalize them to the MNI space and to obtain the CC mask. Then, these masks were averaged to obtain the CC probability map. The value of each voxel in this map could be considered as the probability that the voxel was part of the CC. The above method was also used to create the genu, body, and splenium probability maps.

\section{Diffusion Indices of the CC and Its Subregions}

After the CC probability map was created, the following steps were performed to calculate diffusion indices inside the CC: First, the $b=0$ images were normalized to the MNI space to obtain the transformation parameters. Second, the transformation parameters were used to normalize the corresponding diffusion indices maps (FA, MD, $\lambda_{1}$, and $\lambda_{23}$ ). Then the weighted means of the diffusion indices inside the $\mathrm{CC}$ were estimated by superimposing the probability map on the normalized diffusion-index images.

For patients with CIS, we analyzed the diffusion properties in the NACC. To this end, we normalized lesion images to the MNI space to obtain the lesion distribution in this space. Lesions were then used as masks to calculate the diffusion indices within the NACC. The same method was also used with the genu, body, and splenium probability maps to obtain the diffusion indices inside subregions of the NACC.

\section{Statistical Analysis}

Statistical analysis was performed with the Statistical Package for the Social Sciences, Version 11.5 for Windows (SPSS, Chicago, Illinois). The group comparison for the CC size was performed by using a 2 -sample $t$ test. Repeated-measures analysis of variance was used to assess the effects of group (controls-versus-patients), subregions (genu, body, and splenium), and their possible interactions on diffusion indices. Then a post hoc $t$ test was used to examine the group differences for each subregion when group effects were significant. Correlations between these diffusion indices and EDSS or the area of $\mathrm{CC}$ or T2 lesion volumes were evaluated. A $P$ value $<.01$ was considered statistically significant, and a value between .01 and .05 was considered a trend. ${ }^{37}$

\section{Results}

\section{Comparison of Patients with CIS and Controls in the Entire CC}

One example of the entire CC and its subregions in the midsagittal plane is shown in Fig $1 A$. The average midsagittal CC area was $6.27 \pm 0.60 \mathrm{~cm}^{2}$ for healthy subjects and $5.50 \pm 0.64$ $\mathrm{cm}^{2}$ for patients with CIS. The 2 -sample $t$ test revealed a significant difference in the midsagittal CC area between groups (2-paired, $P=.001$; Fig $1 B$ ). The group difference remained statistically significant after correction for possible influences from individual brain size (normalized CC size: $0.046 \pm 0.005$ for controls and $0.041 \pm 0.004$ for patients with CIS; $P=.001$; Fig $1 C$ ). For diffusion measures, patients with CIS had significantly higher MD and $\lambda_{23}$ and a lower FA in the entire CC with a trend toward a higher $\lambda_{1}(P=.001$ for FA, $P=.004$ for MD, $P=.003$ for $\lambda_{23}, P=.010$ for $\lambda_{1}$; Table) compared with healthy subjects (Table).

\section{Comparison of Patients with CIS and Controls in Subregions of the CC}

Fiber tracking was able to reconstruct callosal fiber bundles projecting into the cortical hemispheres for all healthy subjects. According to their cortical projections, 3 subregions (genu, body, and splenium) of the CC were obtained. Figure 2 shows the entire CC and its 3 subregion probabilistic maps created in the template (MNI) coordinates. These probabilistic maps of the CC are well-defined at the core with higher probabilities, while they become more dispersed and have lower probabilities as they approach cortical regions.

Repeated-measures analysis of variance showed significant group and region effects without any interaction (group-byregion) effects. Therefore, a post hoc $t$ test was performed with group as the independent variable, and FA, MD, $\lambda_{1}$, and $\lambda_{23}$ within the genu, body, and splenium as dependent variables. Compared with healthy subjects, patients with CIS had a significantly lower FA in the body with a trend toward a lower FA in the genu and splenium $(P=.002$ for the body, $P=.011$ for the genu, $P=.019$ for splenium). For MD, CIS had a significantly higher MD in the body and splenium without a difference in the genu $(P=.007$ for the body, $P=.004$ for the 


\begin{tabular}{|c|c|c|c|}
\hline \multicolumn{4}{|c|}{$\begin{array}{l}\text { Comparison of diffusion indices measured in subregions of CC of } \\
\text { NC and CIS }\end{array}$} \\
\hline & NC $(n=19)$ & CIS $(n=19)$ & \multirow[b]{2}{*}{$P^{\mathrm{a}}$} \\
\hline & Mean \pm SD & Mean \pm SD & \\
\hline \multicolumn{4}{|l|}{$\overline{F A}$} \\
\hline Whole CC & $0.46 \pm 0.03$ & $0.42 \pm 0.04$ & .001 \\
\hline Genu & $0.458 \pm 0.038$ & $0.419 \pm 0.053$ & .011 \\
\hline Body & $0.442 \pm 0.032$ & $0.399 \pm 0.046$ & .002 \\
\hline Splenium & $0.494 \pm 0.034$ & $0.453 \pm 0.062$ & .020 \\
\hline \multicolumn{4}{|l|}{$M D^{\mathrm{b}}$} \\
\hline Whole CC & $0.90 \pm 0.06$ & $1.02 \pm 0.16$ & .004 \\
\hline Genu & $0.868 \pm 0.062$ & $0.937 \pm 0.142$ & .064 \\
\hline Body & $0.868 \pm 0.070$ & $0.995 \pm 0.182$ & .009 \\
\hline Splenium & $0.948 \pm 0.078$ & $1.087 \pm 0.181$ & .005 \\
\hline \multicolumn{4}{|l|}{$\lambda_{1}{ }^{\mathrm{c}}$} \\
\hline Whole CC & $1.38 \pm 0.06$ & $1.49 \pm 0.15$ & .010 \\
\hline Genu & $1.333 \pm 0.059$ & $1.417 \pm 0.175$ & .228 \\
\hline Body & $1.310 \pm 0.087$ & $1.409 \pm 0.177$ & .024 \\
\hline Splenium & $1.505 \pm 0.086$ & $1.633 \pm 0.197$ & .016 \\
\hline \multicolumn{4}{|l|}{$\lambda_{23}{ }^{\mathrm{d}}$} \\
\hline Whole CC & $0.66 \pm 0.07$ & $0.79 \pm 0.16$ & .003 \\
\hline Genu & $0.635 \pm 0.071$ & $0.718 \pm 0.151$ & .040 \\
\hline Body & $0.648 \pm 0.070$ & $0.785 \pm 0.188$ & .007 \\
\hline Splenium & $0.670 \pm 0.082$ & $0.814 \pm 0.183$ & .004 \\
\hline
\end{tabular}

${ }^{a}$ A $P$ value $<.01$ was considered statistically significant, while a value between .01 and .05 was considered a trend.

${ }^{\mathrm{b}} \mathrm{MD}=\times 10^{-3} \mathrm{~mm}^{2} / \mathrm{s}$

$\lambda_{1}=\times 10^{-3} \mathrm{~mm}^{2} / \mathrm{s}$

${ }^{\mathrm{d}} \lambda_{23}=\times 10^{-3} \mathrm{~mm}^{2} / \mathrm{s}$

splenium, $P=.060$ for the genu) (Table). For $\lambda_{1}$, CIS had a trend toward being higher in the body and splenium with no difference in the genu $(P=.022$ for the body, $P=.014$ for the splenium, $P=.225$ for the genu) (Fig $3 A$ ). For $\lambda_{23}$, CIS had a significantly higher $\lambda_{23}$ in the body and splenium with a trend toward being higher in the genu $(P=.005$ for the body; $P=$ .003 for the splenium; $P=.037$ for the genu) (Fig 3B).

\section{Cross-Correlation between MR Imaging Parameters Measured in the Entire CC}

The mean lesion volume was $6.12 \pm 9.90 \mathrm{~mL}$ (range, $0.14-$ $33.05 \mathrm{~mL}$ ) in patients with CIS. There was a trend toward inverse correlation between FA from the entire CC and T2 lesion volumes, with a trend toward a positive correlation between MD, $\lambda_{1}$ and $\lambda_{23}$, and T2 lesion volumes $(r=-0.502$, $P=.029$ for FA; $r=0.554, P=.014$ for MD; $r=0.504, P=$ .028 for $\lambda_{1} ; r=0.542, P=.016$ for $\left.\lambda_{23}\right)$.

\section{Discussion}

We used diffusion tensor group tractography to investigate abnormal diffusion within the entire CC and its segments in patients with CIS. First, fiber tracking was performed in each healthy subject with DTIStudio, and the entire CC and its subregions were reconstructed by using the multiple regionof-interest method. Second, the white matter fiber tracts (the entire CC and its subregions) were normalized to the MNI space, and then the probability maps of the entire CC and its subregions were generated by averaging the normalized fiber tracts from all healthy subjects. Then, the probability maps were used as weighted template images to evaluate the average diffusion indices of healthy subjects and patients with CIS within the entire CC and its segments. There are several reasons for applying the diffusion tensor group tractography method. First, in diseased brains, DTI measures are abnormal in normal-appearing brain tissue, especially in the lesions, ${ }^{17,18}$ which might cause unreliable and erroneous termination of tractography. However, the diffusion tensor group tractography is not affected by abnormal tissues. Second, this method is capable of defining the cores of fiber tracts from the healthy population, and the resultant probability maps can then be used as references for studying abnormal diffusion within tracts in neurodegenerative diseases. ${ }^{15,16,19}$ Therefore, in our study, diffusion tensor group tractography was used to evaluate CC integrity and patterns of CC abnormalities in CIS.

In this study, we found that both the volume and microstructure of the CC appear to be damaged at the CIS stage. In the midsagittal CC area, the CC size was reduced relative to healthy subjects (Fig $1 B$ and $1 C$ ), which indicated that the onset of CC atrophy might appear at this stage of CIS, the earliest clinical expression of MS. This result was consistent with previous observations in MS, which reported that the midsagittal CC areas were significantly reduced in patients with MS and that severe atrophy of the CC occurs in longstanding MS., ${ }^{2,3}$ Although our results contradicted those in 1 report in which no atrophy of the CC for CIS was found by the region-of-interest method with MR imaging and diffusionweighted imaging, ${ }^{38}$ the reason might be attributed to the different sample, imaging, and analysis methods.

We found that patients with CIS had a significantly higher MD and $\lambda_{23}$ and a lower FA, with a trend toward higher $\lambda_{1}$ in the entire CC compared with healthy controls (Table). These findings indicated that patients with CIS have abnormal diffusion in the CC, which suggests that CC integrity might be damaged in CIS. The underlying pathologies may be inflammation, gliosis, demyelination, and axonal loss. The analysis of the diffusion tensor eigenvalues may provide more information about pathology. The $\lambda_{23}$ reflects the changes of axonal membrane, myelin sheath, extracellular space, and so forth. ${ }^{28,39-41}$ Loss of axonal structures may lead to the lessrestricted diffusion perpendicular to the main direction of fibers and increased $\lambda_{23}{ }^{28,39-41}$ In our study, we also found that CIS had a trend toward being higher on $\lambda_{1}$, which might be caused by inflammation and gliosis in our patients with CIS who were experiencing the first attack. In combination, these effects are expected to add up to a reduced FA and increased $\mathrm{MD}$ in the entire CC.

To further investigate the patterns of the CC abnormalities in CIS, we divided the entire CC into 3 subregions (genu, body, and splenium) according to their fiber projections, then we created probability maps, and finally we evaluated the diffusion indices inside these subregions on the basis of their probability maps. Statistical analysis revealed that patients with CIS had a significantly lower FA in the body with a trend toward lower FA in the genu and splenium, a significantly higher MD in the body and splenium with no difference in genu, a trend toward higher $\lambda_{1}$ in the body and splenium with no difference in the genu, and a significantly higher $\lambda_{23}$ in the body and splenium with a trend toward higher $\lambda_{23}$ in genu (Table; Fig 3). These results suggested that the subregions of CC were not equally affected, and the severity of abnormalities in the genu was milder than that in the body and splenium in CIS.

Before attempting to explain these findings in relation to 

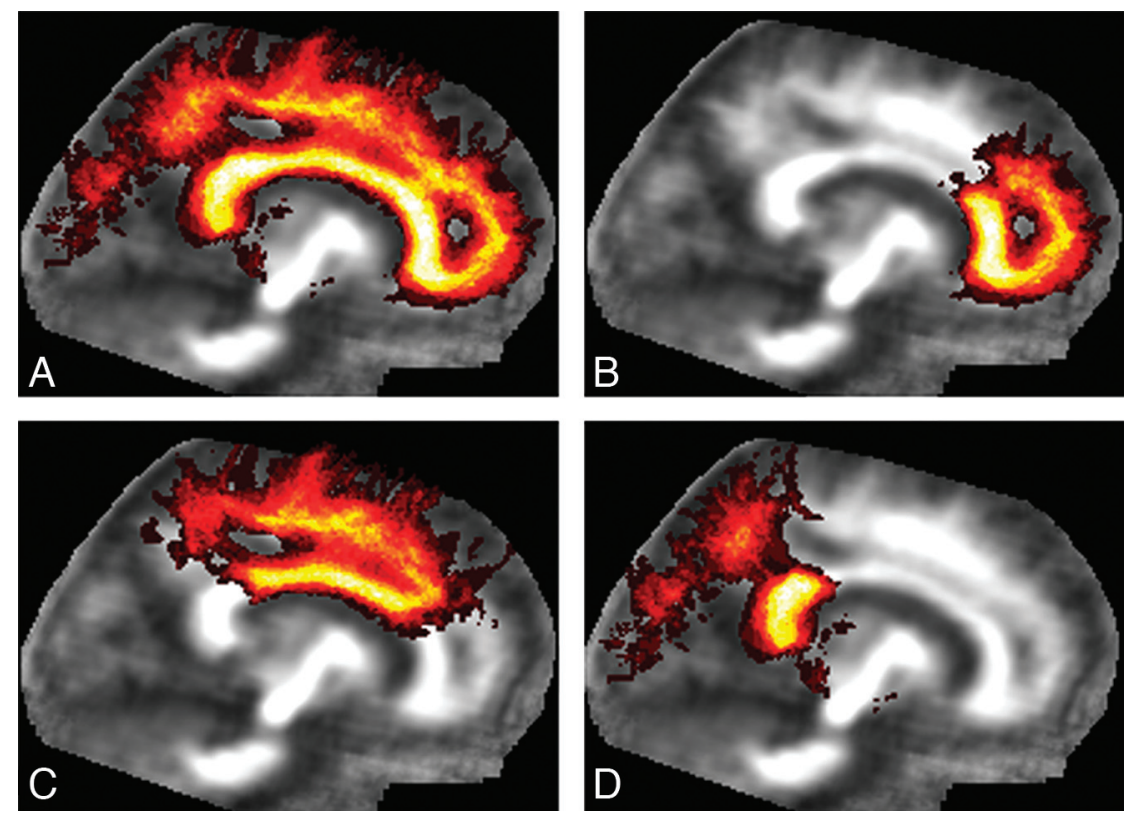

Fig 2. The probability maps for the entire CC and its subregions. $A$, The entire CC probability map. $B$, The genu probability map. $C$, The body probability map. $D$, The splenium probability map. The gray-scale overlay indicates the probability of a voxel being part of the $\mathrm{CC}$ or its subregions.
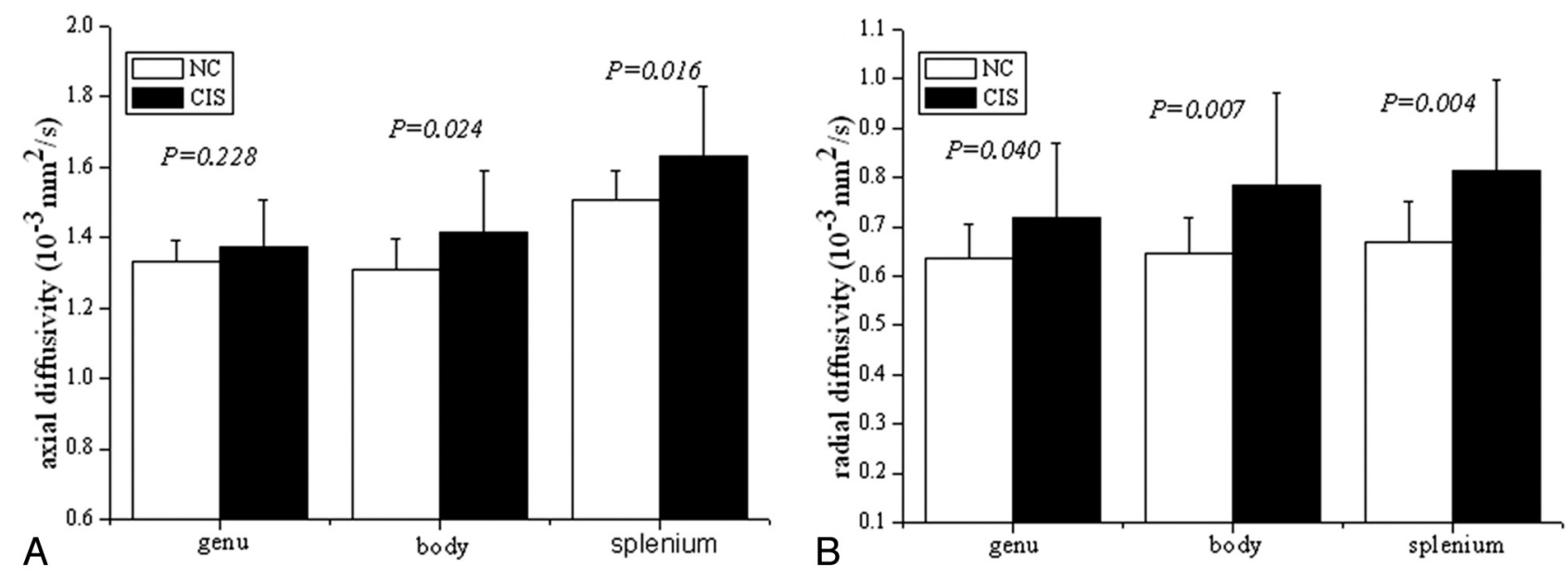

Fig 3. The $\lambda_{1}$ and $\lambda_{23}$ distributions for the 3 subregions of the CC in CIS suggestive of MS and NCs. $A, \lambda_{1}\left(\times 10^{-3} \mathrm{~mm}^{2} / \mathrm{s}\right)$ distribution for the 3 subregions of the CC for these 2 groups. $B, \lambda_{23}\left(\times 10^{-3} \mathrm{~mm}^{2} / \mathrm{s}\right)$ distribution for the 3 subregions of the CC for these 2 groups.

CIS, we interpreted our regional diffusion anisotropy of the $\mathrm{CC}$ in healthy subjects and patients with CIS. In our study, we observed that the CC regional FA was heterogeneous in healthy controls and patients with CIS: FA(splenium) $>$ $\mathrm{FA}$ (genu) $>\mathrm{FA}$ (body), which has also been observed in other studies. ${ }^{7,42,43}$ Some researchers found that tensor anisotropy was significantly larger in the splenium compared with the

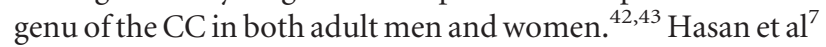
also reported similar FA distribution patterns inside the CC in healthy subjects and patients with relapsing-remitting MS by segmenting the midsagittal CC into 7 subregions. This characterization of FA distribution patterns reflects the differences in the architecture of subregions of the CC. Some previous studies revealed that the interfiber distance in the splenium is smaller than that in the genu and that the body has a greater interfiber distance compared with the genu. ${ }^{20,21}$

In the present study, we observed that the severity of ab- normalities in the genu was milder than that in the body and splenium, which indicated that the subregions of the CC were not equally damaged in patients with CIS. These patterns of CC abnormalities have also been partly observed in MS. ${ }^{7,44,45}$ Ciccarelli et $\mathrm{al}^{44}$ reported that the FA was significantly decreased in the splenium, while it was normal in the genu in patients with MS compared with controls. Evangelou et al ${ }^{45}$ described spatially dependent axonal attenuation loss in the NAWM of the CC in patients with MS. They found a significant decrease in the callosal axonal attenuation in the body of the NAWM of the CC and correlation between the loss in axonal attenuation and regional lesion load. Our studies indicated that the reduced FA observed in the body might reflect reduced axonal attenuation in CIS. Hasan et $\mathrm{al}^{7}$ observed a significant reduction in FA of the body, with a trend toward lower FA in the genu, with no difference in the splenium. The contradictions between our findings in the splenium of the CC 
and the results of Hasan et al might be attributed to different sample and analysis methods.

The diffusion indices derived from the entire NACC correlated well with lesion volume, which might suggest that axonal degeneration resulted in the abnormal diffusion in the CC of our group of patients with CIS. Several previous studies have found some measurements of axonal attenuation correlated with lesion volume, and they used these correlations as evidence for the predominance of wallerian degeneration. ${ }^{45}$ In our study, there were no correlations between any of diffusion indices from the CC and either EDSS scores or disease duration. This might be because that the subject sample is not large enough.

Some possible limitations should be noted in this study. First, although diffusion tensor group tractography was used to create tract probability maps to reduce the effects from lesions in diseased brains, some influences from lesions for the quality of registration cannot completely be excluded. Second, the relatively poor resolution of source images might slightly influence our measurements, so a higher resolution MR imaging scanner might improve the reliability of diffusion measurements. Additionally, the EDSS scores of some patients with CIS were evaluated during the first attack, which may have led to EDSS scores being higher than those in other studies.

\section{Conclusions}

In this article, by using diffusion tensor group tractography, we found the presence of atrophy and abnormal diffusion inside the CC in patients with CIS and found that the severity of damage in the genu was milder than that in the body and splenium. Moreover, we also found the diffusion indices of the entire NACC correlated with T2 lesion load, which indicates that both T2 lesion load and CC atrophy contribute to the damage in CIS. The underlying pathologies might be myelin disease and edema at the stage of CIS. Our findings suggested that atrophy and abnormal diffusion inside the CC appear at the earliest stage of MS, especially in CIS, which indicates that the CC is an early site of abnormal white matter diffusion in CIS and it might be used as a biomarker to monitor the disease progression in CIS and predict the clinical outcome of CIS. Our results also suggested that the pattern of CC abnormalities is spatial heterogeneity in CIS. In conclusion, this quantitative measurement approach of diffusion tensor group tractography can detect occult pathologic abnormalities beyond macroscopic lesions in fiber tracts in CIS and can provide further anatomic insights into mechanisms of white matter damage. Additionally, it might also be used to monitor the specific neurologic deficits in CIS. This method can also be used to investigate abnormal diffusion in other fiber tracts in a variety of neurologic and psychiatric disorders.

\section{References}

1. Barnard RO, Trigg M. Corpus callosum in multiple sclerosis. J Neurol Neurosurg Psychiatry 1974;37:1259-64

2. Simon JH, Schiffer RB, Rudick RA, et al. Quantitative determination of MSinduced corpus callosum atrophy in vivo using MR imaging. AJNR Am J Neuroradiol 1987;8:599-604
3. Dietemann JL, Beigelman C, Rumbach L, et al. Multiple sclerosis and corpus callosum atrophy: relationship of MRI findings to clinical data. Neuroradiology 1988;30:478-80

4. Coombs BD, Best A, Brown MS, et al. Multiple sclerosis pathology in the normal and abnormal appearing white matter of the corpus callosum by diffusion tensor imaging. Mult Scler 2004;10:392-97

5. Rovaris M, Gass A, Bammer R, et al. Diffusion MRI in multiple sclerosis. Neurology 2005;65:1526-532

6. Ge Y, Law M, Johnson G, et al. Preferential occult injury of corpus callosum in multiple sclerosis measured by diffusion tensor imaging. J Magn Reson Imaging 2004;20:1-7

7. Hasan KM, Gupta RK, Santos RM, et al. Diffusion tensor fractional anisotropy of the normal-appearing seven segments of the corpus callosum in healthy adults and relapsing-remitting multiple sclerosis patients. J Magn Reson Imaging 2005;21:735-43

8. Rueda F, Hygino LC Jr, Domingues RC, et al. Diffusion tensor MR imaging evaluation of the corpus callosum of patients with multiple sclerosis. $\mathrm{Arq} \mathrm{Neu}$ ropsiquiatr 2008;66:449-53

9. Gean-Marton AD, Vezina LG, Marton KI, et al. Abnormal corpus callosum: a sensitive and specific indicator of multiple sclerosis. Radiology 1991;180: 215-21

10. Pelletier J, Suchet L, Witjas T, et al. A longitudinal study of callosal atrophy and interhemispheric dysfunction in relapsing-remitting multiple sclerosis. Arch Neurol 2001;58:105-11

11. Miller D, Barkhof F, Montalban X, et al. Clinically isolated syndromes suggestive of multiple sclerosis. Part I. Natural history, pathogenesis, diagnosis, and prognosis. Lancet Neurol 2005;4:281-88

12. Brex PA, Ciccarelli O, O'Riordan JI, et al. A longitudinal study of abnormalities on MRI and disability from multiple sclerosis. N Engl J Med 2002;346:158-64

13. Bester M, Heesen C, Schippling S, et al. Early anisotropy changes in the corpus callosum of patients with optic neuritis. Neuroradiology 2008;50:549-57

14. Yu CS, Lin FC, Liu Y, et al. Histogram analysis of diffusion measures in clinically isolated syndromes and relapsing-remitting multiple sclerosis. Eur J Radiol 2008;68:328-34

15. Lin F, Yu C, Jiang T, et al. Diffusion tensor tractography based-group mapping of the pyramidal tract in relapsing-remitting multiple sclerosis patients. AJNR Am J Neuroradiol 2007;28:278-82

16. Pagani E, Filippi M, Rocca MA, et al. A method for obtaining tract-specific diffusion tensor MRI measurements in the presence of disease: application to patients with clinically isolated syndromes suggestive of multiple sclerosis. Neuroimage 2005;26:258-65

17. Filippi M, Cercignani M, Inglese M, et al. Diffusion tensor magnetic resonance imaging in multiple sclerosis. Neurology 2001;56:304-11

18. Griffin CM, Chard DT, Ciccarelli O, et al. Diffusion tensor imaging in early relapsing-remitting multiple sclerosis. Mult Scler 2001;7:290-97

19. Hua K, Zhang J, Wakana S, et al. Tract probability maps in stereotaxic spaces: analyses of white matter anatomy and tract-specific quantification. Neuroimage 2008;39:336-47

20. Aboitiz F, Scheibel AB, Fisher RS, et al. Fiber composition of the human corpus callosum. Brain Res 1992;598:143-53

21. Lamantia AS, Rakic P. Cytological and quantitative characteristics of four cerebral commissures in the rhesus monkey. J Comp Neurol 1990;291:520-37

22. Witelson SF. Hand and sex differences in the isthmus and genu of the human corpus callosum: a postmortem morphological study. Brain 1989;112(pt 3): 799-835

23. Alexander AL, Lee JE, Lazar M, et al. Diffusion tensor imaging of the corpus callosum in autism. Neuroimage 2007;34:61-73

24. Kochunov P, Thompson PM, Lancaster JL, et al. Relationship between white matter fractional anisotropy and other indices of cerebral health in normal aging: tract-based spatial statistics study of aging. Neuroimage 2007;35: $478-87$

25. Shimony JS, Burton H, Epstein AA, et al. Diffusion tensor imaging reveals white matter reorganization in early blind humans. Cereb Cortex 2006;16: 1653-61

26. Sullivan EV, Adalsteinsson E, Pfefferbaum A. Selective age-related degradation of anterior callosal fiber bundles quantified in vivo with fiber tracking. Cereb Cortex 2006;16:1030-39

27. Kurtzke JF. Rating neurological impairment in multiple sclerosis: an expanded disability status scale (EDSS). Neurology 1983;33:1444-52

28. Song SK, Yoshino J, Le TQ, et al. Demyelination increases radial diffusivity in corpus callosum of mouse brain. Neuroimage 2005;26:132-40

29. Basser PJ, Mattiello J, LeBihan D. MR diffusion tensor spectroscopy and imaging. Biophys J 1994;66:259-67

30. Basser PJ, Pierpaoli C. Microstructural and physiological features of tissues elucidated by quantitative diffusion tensor MRI. J Magn Reson B 1996;111: 209-19

31. Jiang H, van Zijl PC, Kim J, et al. DTIStudio: resource program for diffusion tensor computation and fiber bundle tracking. Comput Methods Programs Biomed 2006;81:106-16. Epub 2006 Jan 18

32. Mori S, Crain BJ, Chacko VP, et al. Three dimensional tracking of axonal 
projections in the brain by magnetic resonance imaging. Ann Neurol 1999;45: 265-69

33. Mori S, Kaufmann WE, Davatzikos C, et al. Imaging cortical association tracts in the human brain using diffusion-tensor-based axonal tracking. Magn Reson Med 2002;47:215-23

34. Stieltjes B, Kaufmann WE, van Zijl PC, et al. Diffusion tensor imaging and axonal tracking in the human brainstem. Neuroimage 2001;14:723-35

35. Conturo TE, Lori NF, Cull TS, et al. Tracking neuronal fiber pathways in the living human brain. Proc Natl Acad Sci U S A 1999;96:10422-27

36. Wakana S, Jiang H, Nagae-Poetscher LM, et al. Fiber tract-based atlas of human white matter anatomy. Radiology 2004;230:77-87

37. Nijeholt GJ, van Walderveen MA, Castelijns JA, et al. Brain and spinal cord abnormalities in multiple sclerosis: correlation between MRI parameters, clinical subtypes and symptoms. Brain 1998;121:687-97

38. Ranjeva JP, Pelletier J, Confort-Gouny S, et al. MRI/MRS of corpus callosum in patients with clinically isolated syndrome suggestive of multiple sclerosis. Mult Scler 2003;9:554-65
39. Le Bihan D. Looking into the functional architecture of the brain with diffusion MRI. Nat Rev Neurosci 2003;4:469-80

40. Pierpaoli C, Basser PJ. Toward a quantitative assessment of diffusion anisotropy. Magn Reson Med 1996;36:893-906

41. Kobayashi S, Hasegawa S, Maki T, et al. Retrograde degeneration of the corticospinal tract associated with pontine infarction. J Neurol Sci 2005;236:91-93

42. Abe $\mathrm{O}$, Aoki S, Hayashi $\mathrm{N}$, et al. Normal aging in the central nervous system: quantitative MR diffusion-tensor analysis. Neurobiol Aging 2002;23:433-41

43. Chepuri NB, Yen YF, Burdette JH, et al. Diffusion anisotropy in the corpus callosum. AJNR Am J Neuroradiol 2002;23:803-08

44. Ciccarelli O, Werring DJ, Barker GJ, et al. A study of the mechanisms of normal-appearing white matter damage in multiple sclerosis using diffusion tensor imaging-evidence of wallerian degeneration. J Neurol 2003;250:287-92

45. Evangelou N, Konz D, Esiri MM, et al. Regional axonal loss in the corpus callosum correlates with cerebral white matter lesion volume and distribution in multiple sclerosis. Brain 2000;123(pt 9):1845-49 